\title{
MOTIVOS PARA EL CONSUMO DE CIBERSEXO Y SU RELACIÓN CON EL GRADO DE SEVERIDAD
}

\author{
Jesús Castro-Calvo \\ castroj@uji.es \\ Dpto. Psicología Básica, Clínica y Psicobiología. Salusex-Unisexsida. \\ Universitat Jaume I \\ Addictive and Compulsive Behaviours Lab, Institute for Health and Behaviour, University of Luxembourg, Esch- \\ sur-Alzette, Luxembourg \\ Marta García-Barba \\ Dpto. Psicología Básica, Clínica y Psicobiología. Salusex-Unisexsida. \\ Universitat Jaume I \\ Beatriz Gil-Juliá \\ Dpto. Psicología Básica, Clínica y Psicobiología. Salusex-Unisexsida. \\ Universitat Jaume \\ Vicente Morrell-Mengual \\ Dpto. Psicología Evolutiva y de la Educación, \\ Universitat de València, Estudi General \\ Rafael Ballester-Arnal \\ Dpto. Psicología Básica, Clínica y Psicobiología. Salusex-Unisexsida. \\ Universitat Jaume I
}

Fecha de Recepción: 24 Marzo 2018

Fecha de Admisión: 10 Abril 2018

\section{RESUMEN}

La práctica del cibersexo es un comportamiento frecuente que puede desembocar en un patrón de consumo compulsivo, incontrolado y asociado a un deterioro funcional en diversos ámbitos (adicción al cibersexo). Por tanto, conocer los motivos que explicarían que una persona realice esta conducta (de modo recreativo o patológico) es prioritario. Así, este trabajo se plantea el objetivo de analizar la prevalencia de distintos motivos para el consumo de cibersexo, las diferencias en función del género y el vínculo entre estos motivos y los síntomas de consumo problemático. Tomando una muestra de 400 jóvenes (50\% hombres) de entre 18-30 años ( $M=22.2)$, se constata que las motivaciones más frecuentes para el uso de Internet con fines sexuales son "encontrar material con el que masturbarme" (70.8\%) y "relajarme del estrés de mis obligaciones" (44\%). En función del género, se aprecian diferencias entre moderadas y grandes $(V>$.13) en 6 de las 8 motivaciones exploradas. Finalmente, varias de estas motivaciones se mostrarían capaces de predecir el $15 \%$ de varianza del tiempo semanal dedicado al consumo de cibersexo y el $32 \%$ de la puntuación en una escala de severidad. 


\section{MOTIVOS PARA EL CONSUMO DE CIBERSEXO Y SU RELACIÓN CON EL GRADO DE SEVERIDAD}

Palabras clave: cibersexo; motivos; severidad; jóvenes

\section{ABSTRACT}

Reasons for the use of cybersex and its relationship with the degree of severity.

The practice of cybersex is a frequent behavior that can lead to a pattern of compulsive and uncontrolled consumption together with a functional impairment in various areas (addiction to cybersex). Therefore, knowing the reasons that explain this behavior (both recreationally and pathologically) is a priority. The aim of this work was to analyze the prevalence of different reasons for cybersex consumption, their differences according to gender and to explore the link between these motives and the symptoms of problematic use. Evaluating a sample of 400 young people ( $50 \%$ men) between 18-30 years old ( $M=22.2)$, it is found that the most frequent motives for using the Internet for sexual purposes are "finding material with which to masturbate" $(70.8 \%)$ and "relax from the stress of my obligations" (44\%). Depending on the gender, we observe moderate to large differences $\left(V_{\geq} .10\right)$ in 6 out of the 8 motivations explored. Finally, some of these motives predict $15 \%$ of the variance of the weekly time spent on cybersex consumption and $32 \%$ of the scores on a severity scale.

Keywords: cybersex; motives; severity; young people

Este trabajo se ha realizado en parte gracias a la ayuda concedida por la Universitat Jaume I de Castellón (P1.1B2015-82).

\section{INTRODUCCIÓN}

Durante los últimos años, el porcentaje de población que usa Internet (también conocido como ratio de penetración) ha crecido exponencialmente, situándose en torno al 78\% en la Unión Europea (80.6\% en España) (International Telecommunication Union, 2017). Las aplicaciones, utilidades y contenidos online disponibles son innumerables, si bien uno de los que acapara el protagonismo es la pornografía. Algunas cifras ejemplifican su popularidad: se estima que en torno al $13 \%$ de búsquedas en Internet incluye términos sexuales (Ogas \& Gaddam, 2011), que en torno al 35\% de las descargas son de material pornográfico y que es tanta su demanda que la producción y difusión de estos materiales genera entre 1 y 97 billones de dólares de beneficio anuales (el amplio rango se debe a la opacidad que envuelve la industria pornográfica) (Wondracek, Holz, Platzer, \& Kruegel 2010). Según las estadísticas publicadas por «Pornhub» (una de las web pornográficas más visitadas), sus servidores comparten cada año en torno a 3.732 petabytes de material pornográfico, lo que equivale en cifras más aprehensibles a 118 gigabytes de pornografía por segundo (alrededor de 95 películas en alta definición) (Pornhub, 2018). La disponibilidad de múltiples herramientas para el acceso a Internet (ordenadores, tabletas, teléfonos inteligentes, gafas de realidad virtual 0 aumentada, etc.), la facilidad para acceder a contenido sexual online, el presumible anonimato que estas páginas garantizan y su gratuidad hacen de Internet la herramienta idónea donde comenzar a experimentar y explorar la sexualidad (Cooper 1998).

El cibersexo, entendido como "el uso de Internet con objetivos de gratificación sexual" (Cooper \& Griffin-Shelley 2002), comprende toda una serie de actividades que pueden realizarse en solitario o bien interactuando con otros usuarios (Wéry \& Billieux, 2016). Las más frecuentes son las que no requieren del contacto con otros usuarios (como la lectura de textos eróticos, la descarga de imágenes 0 el visionado de películas pornográficas). Su prevalencia varía en función de aspectos como el sexo y la edad de los usuarios. En función del sexo, el consumo de pornografía y de cualquier otra actividad sexual online es inferior en chicas (Ballester-Arnal, Castro-Calvo, Gil-Llario, \& Gil-Julià, 
2016). Respecto a su evolución a lo largo del ciclo vital, su consumo resulta infrecuente hasta los 15 años (Wolak, Mitchell, \& Finkelhor, 2007), momento a partir del cual se produce un incremento exponencial que sitúa su prevalencia entre el 33\%-90\% en jóvenes y adultos (Wéry \& Billieux, 2017). En España, el porcentaje de jóvenes entre los 18 y los 25 años que afirman haber buscado pornografía es del $59 \%$ en chicos y $24.2 \%$ en chicas (Ballester-Arnal y cols. 2016), y este porcentaje asciende al 88\% en personas de entre 60-70 años (Castro-Calvo, Ballester-Arnal, GiménezGarcía, \& Gil-Julià, 2017).

Otra actividad sexual online frecuente consistiría en mantener contacto sexual con otros usuarios (ya sea mediante chat, webcam o el intercambio de imágenes). Su prevalencia oscila entre 9.4$30 \%$ (hombres) y $14.9-34 \%$ (mujeres) (Shaughnessy, Byers, \& Walsh, 2011). El rango donde el contacto sexual con otros usuarios es más frecuente se da entre los 18 y los 24 años, decreciendo pasado este periodo (Daneback, Cooper, \& Månsson, 2005); sin embargo, investigaciones recientes en personas mayores demuestran que la prevalencia de comportamientos como chatear reproduciendo fantasías sexuales o el contacto sexual con otros usuarios a través de webcam repuntan nuevamente con la edad (hasta el $30 \%$ y el $20 \%$ respectivamente) (Castro-Calvo y cols. 2017).

Existe cierto debate en torno a los beneficios y los inconvenientes del sexo en Internet, sobre todo cuando se habla del impacto en el desarrollo psicosexual de los jóvenes y los adolescentes (0'Sullivan, 2014). Entre sus beneficios, algunos investigadores sostienen que el sexo en Internet constituye un medio eficaz para la satisfacción del deseo sexual (Daneback, Sevcikova, Mänsson, \& Ross, 2013), compensar la escasez de conocimientos sobre sexualidad (Smith, 2013), encontrar parejas románticas o sexuales en un entorno seguro (Courtice \& Shaughnessy, 2018) 0 para distraerse del aburrimiento y de los problemas cotidianos (Baltieri y cols., 2015).

En el extremo contrario, el sexo en Internet también podría provocar toda una serie de problemas a sus usuarios que serían consecuencia o bien del tipo de actividades sexuales online realizadas 0 de la manera en la que éstas se realizan. Así, ciertas prácticas sexuales online como el sexting se consideran problemáticas independientemente de que se realicen esporádicamente 0 con frecuencia (Cooper, Quayle, Jonsson, \& Svedin 2016). Por otro lado, prácticas sexuales online mayoritarias (como el uso de pornografía) pueden sobrevenir un problema cuando se realizan de forma abusiva; así, uno de los principales riesgos documentados del sexo en Internet es precisamente la posibilidad de desarrollar un cuadro clínico de adicción al cibersexo. La adicción al cibersexo se define como el "uso excesivo e incontrolado de cibersexo que desemboca en problemas laborales, sociales y personales" (Cooper \& Griffin-Shelley 2002). Según Cooper (1998), los adictos al cibersexo pasarían una cantidad de tiempo exagerada realizándolo, persistirían a pesar de sus consecuencias negativas, no tendrían control sobre su inicio o finalización, negarían su problema y fallarían en el intento por controlar su consumo. Otros síntomas del cuadro clínico consistirían en el uso del cibersexo para regular estados emocionales disfóricos, el desarrollo de craving y de una alta reactividad a estímulos condicionados, la preferencia por estímulos reforzantes a corto plazo frente a estímulos de mayor magnitud a largo plazo («delay discounting»), el desarrollo de tolerancia y de síntomas de abstinencia (Rosenberg, Carnes, \& O’Connor, 2014).

Habida cuenta de los problemas derivados de esta condición, una importante cantidad de trabajos se han dirigido a evaluar los factores predictores de este cuadro clínico. En este sentido, Shaugnessy, Byers, Clowater \& Kalinowski (2014) sostienen que "características individuales -en especial los motivos para realizar la actividad sexual online- pueden determinar en qué medida las personas sufren o no consecuencias negativas derivadas" (p. 1195). Sin embargo, pocos estudios se han detenido a explorar en profundidad cuáles son las razones que llevan a que una persona realice actividades sexuales online y si alguna de ellos se relacionaría con el desarrollo de un patrón de 


\section{MOTIVOS PARA EL CONSUMO DE CIBERSEXO Y SU RELACIÓN CON EL GRADO DE SEVERIDAD}

consumo problemático. En concreto, solo un $25 \%$ de los estudios revisados por Short y cols. (2012) exploraban las razones por las que las personas consumen cibersexo y prácticamente ninguno lo hacía con respecto al vínculo con el consumo abusivo de cibersexo. Habida cuenta esta ausencia de estudios, planteamos este trabajo con tres objetivos: 1) analizar la prevalencia de distintos motivos para el consumo de cibersexo en jóvenes entre 18-30 años, 2) sus diferencias en función del género y 3) explorar el vínculo entre estos motivos y diversos síntomas de consumo problemático, considerando el género una variable mediadora en esta relación.

\section{MÉTODO}

\section{Participantes}

En este estudio han participado un total de 400 jóvenes de entre 18-30 años ( $M=22.17$; $D T=2.91)$. La muestra se balanceó en función del género ( $50 \%$ hombres y $50 \%$ mujeres) y la mayoría afirmó tener pareja (50\%) e identificarse como heterosexuales $(64.9 \%)$. En cuanto a creencias religiosas, el $77 \%$ se consideraban ateos o agnósticos, seguido de creyentes no practicantes (19\%) y practicantes (4.5\%). Finalmente, el $98 \%$ dijo disponer de ordenador propio desde el que acceder a Internet.

\section{Instrumentos}

Durante esta investigación, se exploraron múltiples aspectos del comportamiento sexual online y offline. El tiempo medio para completar todos los cuestionarios fue de 28,65 minutos ( $D T=42,66)$. Para este trabajo que presentamos se analizaron únicamente las siguientes variables:

-Variables sociodemográficas: Ios participantes completaron un cuestionario $\mathrm{Ad}$-Hoc en el que se les preguntaba su género, edad, relación de pareja, orientación sexual, creencias y si disponían de ordenador con acceso a Internet.

-Comportamiento sexual online: el Cuestionario de Prácticas Sexuales Online (CPSO, SalusexUnisexsida, 2016) se empleó para explorar aspectos como el tiempo dedicado a la actividad sexual online (“¿Cuántas horas a la semana pasas conectado a páginas sexuales?”) o las motivaciones para consumir cibersexo (p.e., "relajarme", "animarme", "distraerme", etc.). En concreto, se exploraron las 8 motivaciones más frecuentemente reportadas en otros estudios (Paul \& Shim, 2008). La fiabilidad fue de, 68 .

-Severidad del consumo de cibersexo: esta dimensión se evaluó mediante la adaptación española del Cuestionario de Adicción al Cibersexo (ISST, Ballester-Arnal, Gil-Llario, Gómez-Martínez, \& Gil-Julià, 2010). Compuesto por 25 ítems de respuesta dicotómica (Verdadero/Falso), este instrumento evaluaría el grado en que la conducta sexual online resulta o no problemática. Ballester y cols. (2010) encontraron una fiabilidad de .88 para la escala general y una estabilidad temporal (testretest) de, 82 . En el presente estudio, la fiabilidad fue de, 80 .

\section{Procedimiento}

Los participantes en este estudio fueron reclutados y evaluados a través de ADISEX (http://adiccionalsexo.uji.es/), una plataforma que ofrece a cualquier persona interesada la posibilidad de someterse a una completa evaluación del control de impulsos sexuales. La página es accesible a través de cualquier motor de búsqueda mediante los términos "Adicción al cibersexo", "Adicciones sexuales", "Evaluación de la adicción al cibersexo" o "Tratamiento de la adicción al cibersexo". Con el objetivo de enriquecer el perfil e incrementar el número de usuarios que accedían a la evaluación, se inició a través del sistema de publicidad de Facebook (publicaciones sugeridas) una campaña en la que se solicitaba colaboración en un estudio. Para evitar sesgos, en este anuncio no se mencio- 
nó el objetivo concreto de la investigación (únicamente se especificaba que se trataba de una investigación sobre sexualidad).

\section{Análisis de los datos}

Los datos se analizaron mediante el paquete estadístico SPSS Versión 23.0 y el software G*Power versión 3.1 (cálculo del tamaño del efecto). Se realizaron análisis descriptivos para caracterizar los participantes en términos sociodemográficos y de comportamiento sexual online, analizando posteriormente el papel del género a la hora de modular dicha manifestación. Las diferencias en cuanto a conducta sexual online en función del género se evaluaron mediante pruebas t de Student (variables continuas) y contrastes Chi Cuadrado (variables categóricas). El tamaño del efecto de dichos contrastes se estimó a partir de la $d$ de Cohen y de la $V$ de Cramer. Asimismo, se realizaron dos análisis de regresión lineal jerárquica por pasos para analizar la capacidad predictiva de las motivaciones para el uso de Internet con fines sexuales sobre las dos variables dependientes siguientes: (1) el tiempo semanal dedicado al uso del cibersexo y (2) la severidad del consumo.

\section{RESULTADOS}

De los 400 participantes, el 89,8\% afirmó haber utilizado Internet con fines sexuales. En hombres, este porcentaje superaba el obtenido en mujeres (99\% frente al $80,5 \%)$, con diferencias que alcanzaron la máxima significación y un tamaño del efecto medianos ( $\left.c h i^{2}=37,20 ; p<.001 ; V=, 30\right)$. De ahora en adelante, los análisis se realizaron únicamente con aquellos participantes que sí afirmaron haber utilizado Internet con fines sexuales. Respecto al tiempo semanal invertido en la actividad sexual online, los participantes dedicaban una media de 2,08 horas semanales a utilizar internet con fines sexuales $(D T=5.10)$. Nuevamente, las diferencias en función del género alcanzaron la máxima significación y un tamaño del efecto medio $(t=3,8 ; p<, 001 ; d=, 38)$ : así, mientras los hombres invertían cerca de 3 horas semanales a la práctica del cibersexo $(M=3,04 ; D T=6,47)$, las mujeres apenas dedicaban una hora $(M=1,12 ; D T=2,91)$.

Por lo que se refiere a las motivaciones a la hora de utilizar Internet con fines sexuales (tabla 1), el 70,8\% de los participantes afirmó hacerlo para encontrar material con el que masturbarse, seguido del $47 \%$ que lo hacía para aprender cosas nuevas sobre sexo (p.e., nuevas posturas sexuales) y del $44 \%$ al que le permitía relajarse del estrés de sus obligaciones. En función del género, los hombres mostraban una prevalencia mayor que las mujeres en 6 de las 8 motivaciones exploradas, con tamaños del efecto entre bajos y moderados $(V \geq, 13)$.

Tabla 1. Prevalencia total y en función del género de distintos aspectos del comportamiento sexual online

\begin{tabular}{|c|c|c|c|c|c|}
\hline & Total & Hombres & Mujeres & $\mathrm{Chi}^{2}$ & $V$ \\
\hline \multicolumn{6}{|l|}{ Motivaciones para la práctica del cibersexo } \\
\hline Distraerme & $40,8 \%$ & $50 \%$ & $31,5 \%$ & $14,17 * * *$ & ,18 \\
\hline Encontrar material sexual con el que masturbarme & $70,8 \%$ & $86 \%$ & $55,5 \%$ & $44,95 * * *$ & ,33 \\
\hline Disfrutar actividades sexuales que no hago habitualmente & $24,5 \%$ & $32,5 \%$ & $16,5 \%$ & $13,84 * * *$ & ,18 \\
\hline Aprender cosas sobre sexo & $47 \%$ & $42,5 \%$ & $51,5 \%$ & 3,25 &, 09 \\
\hline Relajarme del estrés de mis obligaciones & $44 \%$ & $50,5 \%$ & $37,5 \%$ & $6,89 * *$ & ,13 \\
\hline Animarme cuando estoy decaído & $13,5 \%$ & $15,5 \%$ & $11,5 \%$ & 1,37 &, 05 \\
\hline Conocer a otras personas & $11,3 \%$ & $16,5 \%$ & $6 \%$ & $11,04 * *$ &, 16 \\
\hline Intentar establecer relaciones & $9 \%$ & $14,5 \%$ & $3,5 \%$ & $14,77 * * *$ &, 19 \\
\hline
\end{tabular}

Nota: ${ }^{*} \mathrm{p}<0.05 ; * * \mathrm{p}<0.01 ; * * * \mathrm{p}<0.001$ 


\section{MOTIVOS PARA EL CONSUMO DE CIBERSEXO Y SU RELACIÓN CON EL GRADO DE SEVERIDAD}

Finalmente, se realizaron dos regresiones lineales jerárquicas para analizar la capacidad predictiva de las motivaciones para el consumo de cibersexo, el género y la interacción entre ambas variables sobre el tiempo semanal dedicado a la práctica del cibersexo y su severidad (tabla 2). En el primer caso, ninguno de los términos de interacción incluidos en la ecuación de regresión (bloque 2) mostró capacidad predictiva significativa sobre el tiempo semanal online con fines sexuales de modo que el modelo final únicamente tuvo en cuenta variables incluidas en el primer bloque $(F=8,16 ; p<, 001)$. En concreto, 2 de las motivaciones exploradas mostraron capacidad predictiva positiva sobre el tiempo semanal dedicado al cibersexo: disfrutar de actividades sexuales que no se realizan con asiduidad $(\beta=1,23)$ y animarse ante estados de ánimo negativos $(\beta=2,78)$. Estas dos variables permitían explicar en torno al $14 \%$ de la varianza del tiempo de uso semanal de cibersexo. En la segunda ecuación de regresión, el modelo más satisfactorio $(F=14,51 ; p<, 001)$ incluyó 2 términos de interacción y 5 variables del primer bloque de la ecuación. Estas 7 variables permitían explicar casi el $37 \%$ de varianza de la severidad del consumo de cibersexo. Concretamente, la severidad predicha por este modelo fue mayor en hombres $(\beta=-1,76)$ y en participantes cuyas motivaciones para el consumo de sexo online tenían que ver con escapar del estrés cotidiano $(\beta=0,85)$, intentar establecer relaciones románticas 0 sexuales con otros usuarios $(\beta=1,71)$ y en general con conocer a otras personas online $(\beta=1,97)$ y con encontrar materiales con los que poder masturbarse $(\beta=2,40)$. En cuanto a los términos de interacción, se aprecia que la capacidad predictiva de dos de las motivaciones sobre la severidad del consumo de cibersexo se multiplicaba en mujeres: así, las que decían emplear Internet a fin de encontrar material con el que masturbarse o para aprender sobre sexo mostraban un mayor incremento de la severidad que los hombres que respondían positivamente a estas mismas motivaciones. La figura 1 ejemplifica la interacción entre el género y una de estas motivaciones.

Tabla 1. Prevalencia total y en función del género de distintos aspectos del comportamiento sexual online

\begin{tabular}{|c|c|c|c|c|c|c|}
\hline & \multicolumn{3}{|c|}{ Tiempo semanal dedicado al cibersexo } & \multicolumn{3}{|c|}{ Severidad del consumo de cibersexo } \\
\hline & $\beta$ & $\mathrm{p}$ & $R^{2}$ & $\beta$ & $\mathrm{p}$ & $R^{2}$ \\
\hline \multicolumn{7}{|l|}{ Bloque 1} \\
\hline Género & $-0,94$ & 07 & $13,9 \%$ & $-1,76$ & $<., 01$ & $34,7 \%$ \\
\hline Distraerme & 0,59 &, 27 & & 0,29 &, 43 & \\
\hline Encontrar material sexual con el que masturbarme & 0,79 &, 16 & & 2,40 & $<, 001$ & \\
\hline Disfrutar actividades sexuales que no hago habitualmente & 1,23 & 03 & & 0,75 & 05 & \\
\hline Aprender cosas sobre sexo & 0,46 &, 35 & & 0,18 &, 58 & \\
\hline Relajarme del estrés de mis obligaciones & 0,18 &, 72 & & 0,85 & 02 & \\
\hline Animarme cuando estoy decaído & 2,78 & $<, 001$ & & 0,81 &, 12 & \\
\hline Conocer a otras personas & 1,83 & 06 & & 1,97 & ,005 & \\
\hline Intentar establecer relaciones & 1,33 & ,233 & & 1,71 & 02 & \\
\hline \multicolumn{7}{|l|}{ Bloque 2} \\
\hline Encontrar material sexual con el que masturbarme $X$ Género & & & NA & 1,93 & ,02 & $36,5 \% *$ \\
\hline Aprender cosas sobre sexo $\mathrm{X}$ Género & & & & 1,50 & 02 & \\
\hline
\end{tabular}

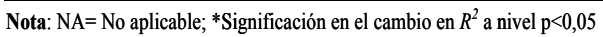


Figura 1. Genero moderando la asociacion entre el uso de Internet para encontrar contenidos con los que masturbarse

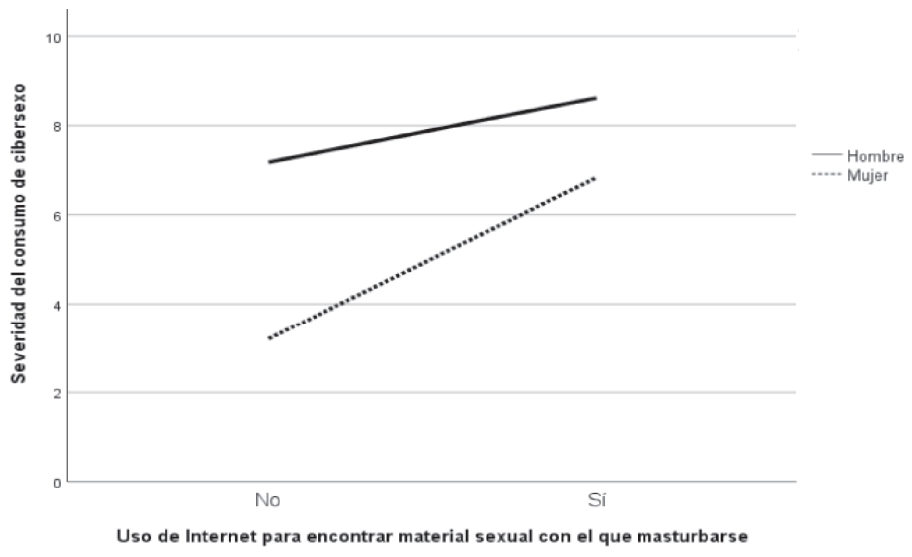

\section{DISCUSIÓN Y CONCLUSIONES}

El principal objetivo de esta investigación ha sido aportar luz sobre uno de los aspectos que podrían ayudar a explicar de forma más satisfactoria el uso y abuso de Internet con fines sexuales: las motivaciones que hay detrás de esta conducta. En concreto, se analizan las ocho razones para el consumo de cibersexo más frecuentemente reportadas en estudios previos, su manifestación en función del género y la capacidad predictiva sobre indicadores clínicos como el tiempo semanal dedicado a la actividad sexual online y su severidad. Teniendo en cuenta que se trataría de un estudio preliminar, en este trabajo se evaluaron únicamente chicos y chicas (400 en total) de entre $18 \mathrm{y}$ 30 años. La razón para escoger una muestra con un rango tan limitado es que los estudios que exploran las motivaciones de acuerdo a la edad demuestran que éstas varían a lo largo del ciclo vital (Castro-Calvo y cols., 2017; Daneback y cols., 2005), de modo que incluir un rango más amplio dificultaría la generalización de resultados. Con todo, este estudio permite extraer tres conclusiones: (1) si bien existen paralelismos entre el perfil de motivaciones para el uso de cibersexo hallado en este estudio y el que se documenta en investigaciones previas, las prevalencias varían notablemente; (2) se confirma que las motivaciones que empujan a hombres y mujeres al consumo de cibersexo son muy diferentes y; (3) que estas motivaciones constituyen (en algunos casos en interacción con el género) buenos predictores del uso (tiempo semanal) y la severidad del consumo de cibersexo.

Como apuntábamos, el análisis de las motivaciones que explican el consumo de cibersexo muestra ciertas incongruencias con el patrón reportado en la literatura. Así, mientras que la distracción es una de las razones a las que se alude con más frecuencia en los estudios internacionales (Cooper y cols., 2002; Wéry \& Billieux, 2016), en nuestro estudio apenas un 40\% afirmó consumir cibersexo por esta razón. En su lugar, la motivación esgrimida con más frecuencia $(70,8 \%)$ fue encontrar contenidos sexuales con los que masturbarse. Este hallazgo validaría el modelo de motivaciones recientemente propuesto por Grubbs y cols. (2017), que explicaría el uso del cibersexo a partir lo que denominan "motivaciones hedónicas" y que se resumiría en la búsqueda rápida de la satisfacción sexual a partir de prácticas y actividades sexuales que impliquen una inversión relativamente pequeña de tiempo, refuerzo sexual inmediato y ausencia de implicación emocional. En el 


\section{MOTIVOS PARA EL CONSUMO DE CIBERSEXO Y SU RELACIÓN CON EL GRADO DE SEVERIDAD}

extremo contrario, la prevalencia de razones que con frecuencia se asocian a la manifestación de problemas clínicos de adicción al cibersexo (usar Internet con fines sexuales para mejorar el estado de ánimo o fantasear con actividades sexuales que no se suelen realizar offline) (Reid y cols., 2011) resultó ser muy baja.

En función del género, los hombres respondieron afirmativamente en mayor medida que las mujeres a la mayoría de las motivaciones exploradas, en especial al uso de Internet a fin de encontrar material con el que masturbarse (86\% frente al $55 \%)$. Estos resultados son coherentes con los estudios que reportan un mayor consumo de pornografía en hombres (Ballester-Arnal y cols., 2016), la principal actividad sexual online asociada a esta motivación. En cuanto al perfil de motivaciones en mujeres, éstas lo consumen casi a partes iguales para la búsqueda de contenidos explícitos con los que masturbarse y para aprender cosas sobre sexo, lo que demuestra que en su caso las razones educativas tienen casi tanto valor como la propia búsqueda del placer.

Para terminar, dos de las motivaciones clásicamente asociadas al consumo problemático de cibersexo (uso del cibersexo a fin de mejorar el estado del ánimo o para fantasear con nuevas prácticas sexuales) explican el $14 \%$ de la varianza del tiempo semanal dedicado al cibersexo. Sin embargo, estas motivaciones no alcanzan luego la significación a la hora de predecir la severidad del consumo de cibersexo, lo que sugeriría que tal vez estén más relacionadas con una mayor implicación en términos de dedicación temporal que de manifestación patológica o disfuncional. Las que si mostraron relación con la severidad fueron el género (ser hombre), dos motivaciones sociales (uso de Internet para conocer a gente e intentar establecer relaciones románticas o sexuales), una hedónica (encontrar material para masturbarse) y una de regulación emocional (relajarme del estrés). Además, una de las motivaciones mencionadas (encontrar material con el que masturbarme) junto con el uso del sexo online con fines educativos mostraron una interacción positiva con el género, confirmando que la capacidad de estos motivos para predecir la severidad del consumo de cibersexo se acentúa cuando son mujeres las que los reportan.

\section{REFERENCIAS}

Ballester-Arnal, R., Castro-Calvo, J., Gil-Llario, M. D., \& Gil-Julià, B. (2016). Cybersex Addiction: A Study on Spanish College Students. Journal of Sex \& Marital Therapy, 43(6), 567-584. https://doi.org/10.1080/0092623X.2016.1208700

Ballester-Arnal, R., Gil-Llario, M. D., Gómez-Martínez, S., \& Gil-Julià, B. (2010). Psychometric properties of an instrument for assessing cyber-sex addiction. Psicothema, 22(4), 1048-53.

Baltieri, D. A., Junqueira-Aguiar, A. S., De Oliveira, V. H., De Souza-Gatti, A. L., \& De Souza-Aranha, R. A. (2015). Validation of the Pornography Consumption Inventory in a Sample of Male Brazilian University Students. Journal of Sex and Marital Therapy, 41(6), 649-660. https://doi.org/10.1080/0092623X.2014.958793

Castro-Calvo, J., Ballester-Arnal, R., Giménez-García, C., \& Gil-Julià, B. (2017). Comportamiento sexual online en adultos mayores. International Journal of Developmental and Educational Psychology, 2(1), 89-98.

Cooper, A. (1998). Sexually Compulsive Behavior. Contemporary Sexuality, 32(4), 1-3.

Cooper, A., \& Griffin-Shelley, E. (2002). The internet:The next sexual revolution. In A. Cooper (Ed.), Sex \& the internet: A guidebook for clinicians (pp. 1-15). New York, NY: Brunner-Routledge.

Cooper, A., Morahan-Martin, J., Mathy, R. M., \& Maheu, M. (2002). Toward an increased understanding of user demographics in online sexual activities. Journal of Sex and Marital Therapy, 28(2), 105-129. https://doi.org/10.1080/00926230252851861

Cooper, K., Quayle, E., Jonsson, L., \& Svedin, C. G. (2016). Adolescents and self-taken sexual 
images: A review of the literature. Computers in Human Behavior, 55, 706-716. https://doi.org/10.1016/j.chb.2015.10.003

Courtice, E. L., \& Shaughnessy, K. (2018). The Partner Context of Sexual Minority Women's and Men's Cybersex Experiences: Implications for the Traditional Sexual Script. Sex Roles, 78(3-4), 272-285. https://doi.org/10.1007/s11199-017-0792-5

Daneback, K., Cooper, A., \& Månsson, S.-A. (2005). An Internet Study of Cybersex Participants. Archives of Sexual Behavior, 34(3), 321-328. https://doi.org/10.1007/s10508-005-3120-z

Daneback, K., Sevcikova, A., Mänsson, S.-A., \& Ross, M. W. (2013). Outcomes of using the internet for sexual purposes: fulfilment of sexual desires. Sexual Health, 10(1), 26-31. https://doi.org/10.1071/SH11023

Grubbs, J., Braden, A. L., Kraus, S., Wilt, J., \& Wright, P. J. (2017). Pornography and PleasureSeeking: Toward a Hedonic Reinforcement Model. https://doi.org/10.17605/OSF.IO/YKQ8S

International Telecommunication Union. (2017). Measuring the Information Society Report 2017. Geneva: International Telecommunication Union, ITU.

O'Sullivan, L. F. (2014). Linking online sexual activities to health outcomes among teens. In E. S. Lefkowitz \& S. A. Vasilenko (Eds.), New directions for child and adolescent development (Vol. 2014, pp. 37-51). San Francisco, CA: Wiley Periodicals. https://doi.org/10.1002/cad

Ogas, 0., \& Gaddam, S. (2011). A billion wicked thoughts. New York, NY: Penguin.

Paul, B., \& Shim, J. W. (2008). Gender, sexual affect, and motivations for internet pornography use. International Journal of Sexual Health, 20(3), 187-199. https://doi.org/10.1080/19317610802240154

Pornhub. (2018). Pornhub's 2018 Year in Review. Retrieved February 4, 2018, from https://www.pornhub.com/insights

Reid, R. C., Li, D. S., Gilliland, R., Stein, J. A., \& Fong, T. (2011). Reliability, validity, and psychometric development of the pornography consumption inventory in a sample of hypersexual men. Journal of Sex and Marital Therapy, 37(5), 359-385. https://doi.org/10.1080/0092623X.2011.607047

Rosenberg, K. P., Carnes, P. J., \& O'Connor, S. (2014). Evaluation and treatment of sex addiction. Journal of Sex \& Marital Therapy, 40(2), 77-91. https://doi.org/10.1080/0092623X.2012.701268

Shaughnessy, K., Byers, E. S., Clowater, S. L., \& Kalinowski, A. (2014). Self-appraisals of arousaloriented online sexual activities in university and community samples. Archives of Sexual Behavior, 43(6), 1187-1197. https://doi.org/10.1007/s10508-013-0115-z

Shaughnessy, K., Byers, E. S., \& Walsh, L. (2011). Online Sexual Activity Experience of Heterosexual Students: Gender Similarities and Differences. Archives of Sexual Behavior, 40(2), 419-427.

Short, M. B., Black, L., Smith, A. H., Wetterneck, C. T., \& Wells, D. E. (2012). A Review of Internet Pornography Use Research: Methodology and Content from the Past 10 Years. Cyberpsychology, Behavior, and Social Networking, 15(1), 13-23. https://doi.org/10.1089/cyber.2010.0477

Smith, M. (2013). Youth Viewing Sexually Explicit Material Online: Addressing the Elephant on the Screen. Sexuality Research and Social Policy, 10(1), 62-75. https://doi.org/10.1007/s13178012-0103-4

Wéry, A., \& Billieux, J. (2016). Online sexual activities: An exploratory study of problematic and nonproblematic usage patterns in a sample of men. Computers in Human Behavior, 56(October 2017), 257-266. https://doi.org/10.1016/j.chb.2015.11.046

Wéry, A., \& Billieux, J. (2017). Problematic cybersex: Conceptualization, assessment, and treatment. 


\section{MOTIVOS PARA EL CONSUMO DE CIBERSEXO Y SU RELACIÓN CON EL GRADO DE SEVERIDAD}

Addictive Behaviors, 64, 238-246. https://doi.org/10.1016/j.addbeh.2015.11.007

Wolak, J., Mitchell, K., \& Finkelhor, D. (2007). Unwanted and Wanted Exposure to Online Pornography in a National Sample of Youth Internet Users. PEDIATRICS, 119(2), 247-257. https://doi.org/10.1542/peds.2006-1891

Wondracek, G., Holz, T., Platzer, C., Kirda, E., \& Kruegel, C. (2010). Is the Internet for Porn? An Insight Into the Online Adult Industry. In 9th Workshop on the Economics of Information Security (pp. 1-14). Boston. 